\title{
Liquid Mass Sensing Using Resonating Microplates under Harsh Drop and Spray Conditions
}

\author{
Said Mahajne, ${ }^{1}$ Dafne Guetta, ${ }^{1}$ Stella Lulinsky, ${ }^{2}$ Slava Krylov, ${ }^{2}$ and Yoav Linzon ${ }^{2}$ \\ ${ }^{1}$ Department of Physics and Optical Engineering, Ort Braude College, 21982 Karmiel, Israel \\ ${ }^{2}$ School of Mechanical Engineering, Faculty of Engineering, Tel Aviv University, 69978 Tel Aviv, Israel \\ Correspondence should be addressed to Yoav Linzon; yoavlinzon@tauex.tau.ac.il
}

Received 28 July 2014; Accepted 20 December 2014; Published 31 December 2014

Academic Editor: Sergey B. Mirov

Copyright (c) 2014 Said Mahajne et al. This is an open access article distributed under the Creative Commons Attribution License, which permits unrestricted use, distribution, and reproduction in any medium, provided the original work is properly cited.

\begin{abstract}
We have performed in situ real time mass sensing of deposited liquid volatile droplets and sprays using plate-like microstructures, with robust and reusable performance attained over harsh conditions and multiple cycles of operation. A home-built electrooptical sensing system in ambient conditions has been used. The bimorph effect on the resonant frequency of altered mass loading, elasticity, and strain had been carefully compared, and the latter were found to be negligible in the presence of nonviscous liquids deposited on top of our microplate devices. In resonant mode, the loaded mass has been estimated from measured resonant frequency shifts and interpreted from a simple, uniformly deposited film model. A minimum submicrogram detectable mass was estimated, suggesting the system's potential for robust, fast, and reusable sensing capabilities, in the presence of volatile liquids under harsh operation conditions.
\end{abstract}

\section{Introduction}

Early on in micromachine miniaturization, as silicon-based flexural elements were shrinking, their potential as excellent mass sensors has been recognized [1]. Since then, resonators based on microelectromechanical systems (MEMS) and nanoelectromechanical systems (NEMS) have been proven as excellent platforms for material spectroscopy in a wide range of sensing applications, including solid [2-5], gas [6-10], and biochemical fluid composition [11, 12], with unprecedented potential for large-scale integration of multiplexed sensors in small monolithic packages [13]. At the ultimate sensitivity limit of inertial mass sensing facilitating such resonating devices [14], recent experiments using NEMS resonators have successfully achieved the power to resolve single molecules [3], atoms [4], and even individual protons in real time [15]. Yet, in realistic sensing scenarios nanoscale mechanical elements are still fragile in the out-of-lab tests [16], and microscale devices are widely dominant for durably functioning mechanical sensors in applications.

Typically, NEMS and MEMS resonators (operating in dynamic rather than static mode, in the high-frequency acoustic regime [7]) are driven through closed-gap configuration, and sensing in the gas or liquid phase is limited to clean surface preparation procedures, set by limitations related to stiction, viscous drag, and squeezed-film damping [7, 10$12,16]$. Durable operation under harsh environmental conditions is an important ongoing goal for realistic integrated MEMS/NEMS sensors.

In the present work we have used a single-layer freestanding silicon microplate resonator (MPR) for detection of added liquid mass and attained sustainable operation of the MPR under harsh wet conditions. Mass spectroscopy using the MPR with an optoelectronic detection scheme synchronized in the frequency domain on a pure mechanical motion mode was achieved over long stability periods.

The paper is organized as follows: in Section 2.1 we detail our home-made optoelectronic setup, and in Section 2.2 device fabrication and basic mechanical characterization are described. Section 3 shows results for sensing in both drop and spray deposition conditions, and the corresponding mass spectroscopy limit of our setup is deduced. In Section 4, we finalize the paper with conclusions and prospects for future studies. 


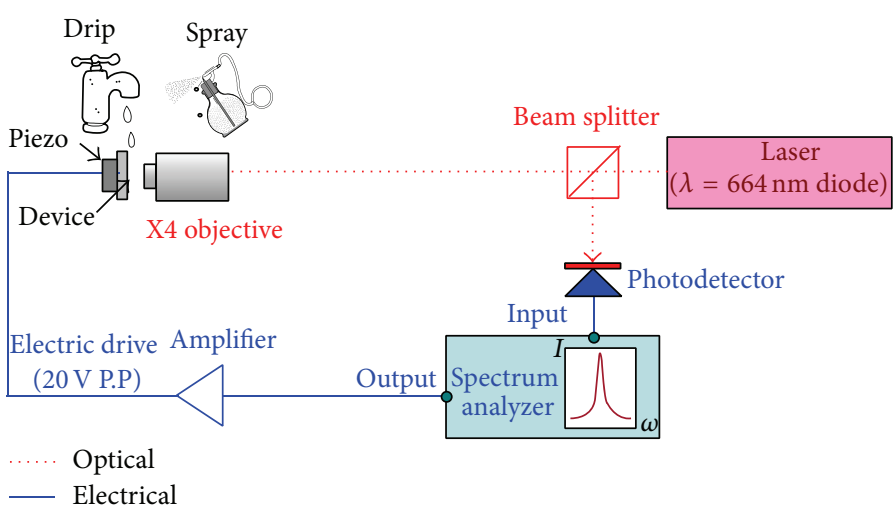

(a)

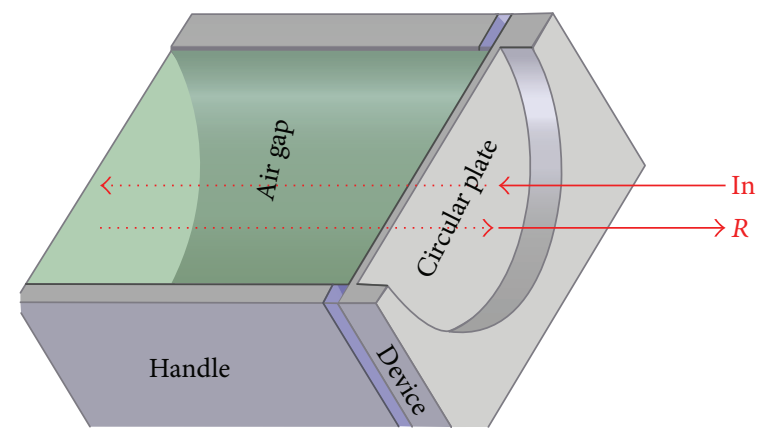

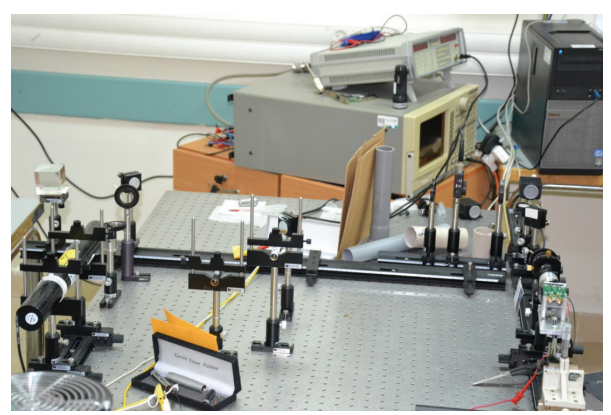

(b)

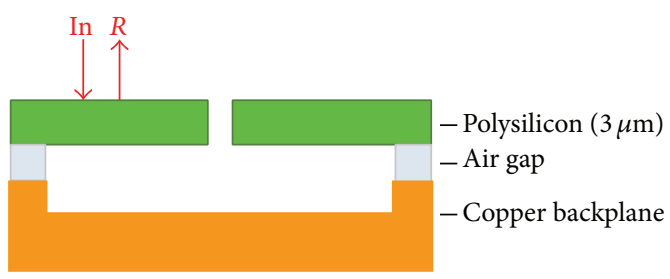

(c)

Figure 1: (a) Schematics of the experimental setup. (b) Photograph of setup. (c) Illustration of cross section (left) and side-view (right) in device structure and light path.

\section{Experimental Section}

2.1. Optoelectronic and Sensing Setup. A schematic of our home-built, free-space, optoelectronic setup is shown in Figure 1(a), and a corresponding photograph is presented in Figure 1(b). Figure 1(c) shows the cross section and side-view of device layers structure and optical light path.

The sensing scheme relies on dynamic actuation [16], where the excitation is electrical via a piezoelectric crystal mounted behind the device chip and motion is monitored optically. Light from a HeNe laser source enters a $4 \mathrm{x}$ microscope objective, which is then back-reflected from the device surface, from which it interferes with the highly reflective backside copper foil (see illustrations in Figure 1(c)). The reflected beam is sent back through a beam splitter into either a CCD camera monitoring the device surface or a photodetector (Thorlabs DET400, 0-20 MHz). During actuation, the reflectance coefficient of the resonator stack vibrates, which produces a resonance signal at the mechanical frequencies, where the signal is proportional to the intensity of the emerging linearly polarized reflected wave [17]. The detector signal is fed into a network analyzer (Stanford Research SR785, 0-102.4 KHz), with a swept frequency detection synchronized with the harmonic actuation signal frequency. Swept $f$-domain measurements thus yield direct amplitude indication.

The mechanical properties of the microplates depend on the temperature, where the resonance frequency is known to decrease somewhat with increasing temperature [17]. As here we aim to measure mass loading rather than thermal effects, we have set environmental temperature constant throughout the experiment $\left(21^{\circ} \mathrm{C} \pm 1^{\circ} \mathrm{C}\right.$ ambient temperature $)$ and have worked with the lowest possible laser power where a signal has been obtained (minimized local heating on the plate surface) and subsequently used the physical parameters appropriate for room temperature operation, in the performance and sensitivity estimations below.

2.2. Mechanical Device Characteristics. Figures 2(a)-2(c) show surface images of our device before and during liquid dropping and following its exposure to spray conditions, respectively. These plates are of radius $500 \mu \mathrm{m}$ and made from n-type highly doped polycrystalline $\mathrm{Si}$ with an upper device layer of $35 \mu \mathrm{m}$, a buried silicon dioxide layer of $4 \mu \mathrm{m}$, and a handle layer single-crystal [100] Si of $400 \mu \mathrm{m}$ thickness (see [18] for fabrication details). In Figure 2(d), a typical widespan response spectrum of the microplate is shown.

Theoretically, considering a plate of radius $a$ centered at the origin and located at the $x-y$ plane, during time $t$ the plate height at a planar observation point $(x, y)$ from its equilibrium is taken to be $u(x, y, t)$. The plate dynamics is governed by the wave equation [19]:

$$
\frac{\partial^{2} u}{\partial t^{2}}=v^{2}\left(\frac{\partial^{2} u}{\partial x^{2}}+\frac{\partial^{2} u}{\partial y^{2}}\right)
$$




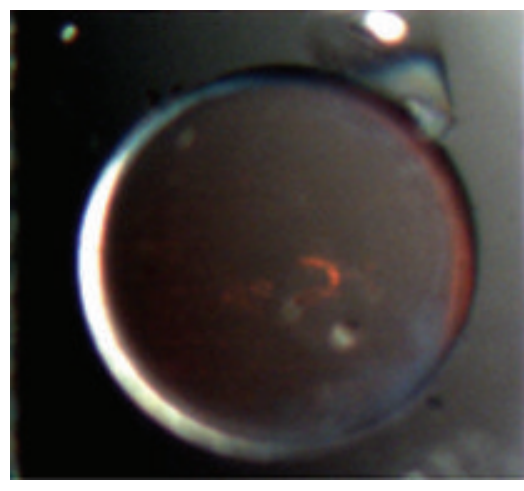

(a)

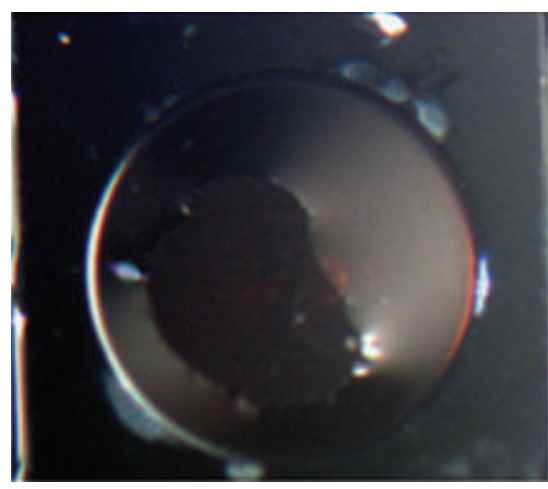

(b)

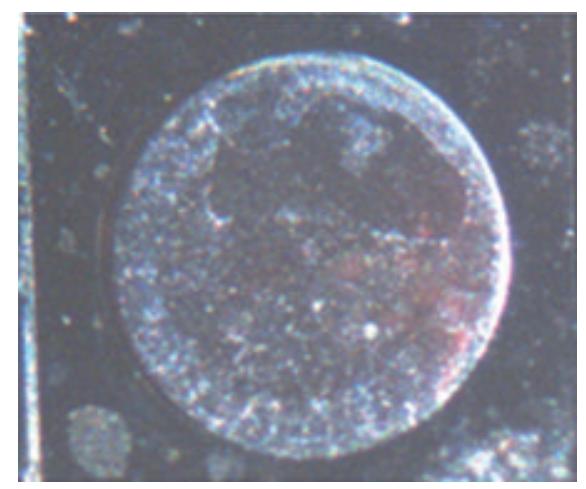

(c)

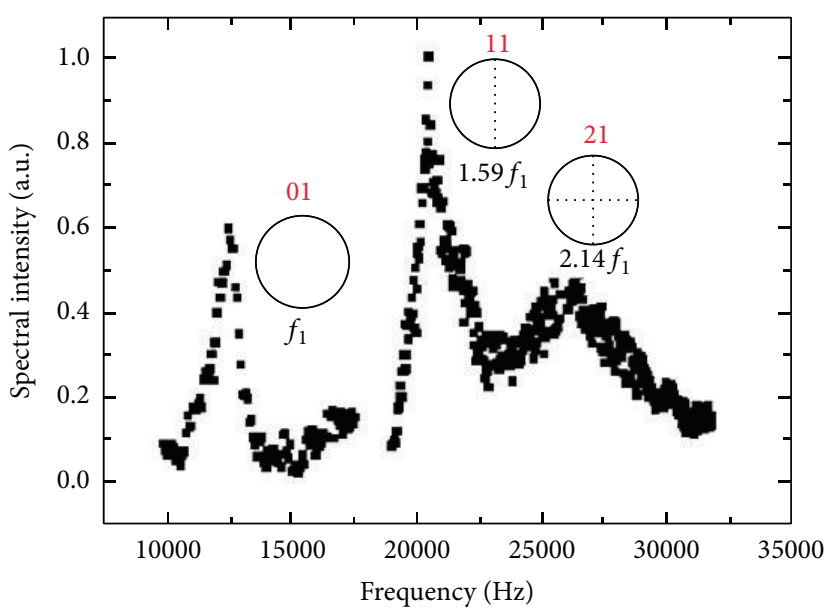

(d)

Figure 2: (a)-(c) Optical images of microplate devices in various phases of the experiment: (a) before sensing cycle; (b) following a harsh drop session (as detailed below); (c) following a harsh spray session (as detailed below). (d) Wide response spectrum of the lowest visible mechanical flexural modes on dry plate.

with the conditions corresponding to clamped edges: $u=0$ at $\sqrt{x^{2}+y^{2}}=a$. The acoustic wave velocity is given by [19]

$$
v=\sqrt{\frac{Y \varepsilon}{\rho}}
$$

where $Y$ is Young's modulus, $\varepsilon$ is the strain, and $\rho$ is the density. Solution of (1) yields an expression for the resonant frequencies of flexural vibration modes:

$$
f_{m n}=\frac{\alpha_{m n}}{2 \pi a} \sqrt{\frac{Y \varepsilon}{\rho}}
$$

with integer mode indices $m=0,1,2,3, \ldots$ and $n=$ $1,2,3, \ldots$, denoting the number of nodes in azimuthal and radial directions, correspondingly, and $\alpha_{m n}$ are proportionality constants, the first of which are [19] $\alpha_{01}=2.404, \alpha_{11}=$ $3.832, \alpha_{21}=5.135, \alpha_{02}=5.52, \alpha_{31}=6.379$, and $\alpha_{12}=7.016$.

In our plates, using a measured fundamental mode frequency of $12.5 \mathrm{KHz}$ (Figure 2(d)) and the physical parameters of polycrystalline $\mathrm{Si}\left(Y=130 \mathrm{GPa}\right.$ and $\rho=2650 \mathrm{~kg} / \mathrm{m}^{3}$ at room temperature) and measured plate radius $a=500 \mu \mathrm{m}$, we estimate the in-built equilibrium strain of $\varepsilon=4 \times 10^{-6}$, which is in agreement with the typical values observed in polycrystalline Si-on-insulator films [20]. Thus we find our considerations, including the neglecting of thermal heating effect, valid.

Using the above value for the in-built strain, we obtain theoretically, using (3), for the higher modes, $f_{11}=$ $(3.832 / 2 \pi a) \sqrt{Y \varepsilon / \rho}=1.59 f_{01}=19.9 \mathrm{KHz}$ and $f_{21}=$ $(5.135 / 2 \pi a) \sqrt{Y \varepsilon / \rho}=2.14 f_{01}=26.7 \mathrm{KHz}$, in agreement with the experimental frequencies in Figure 2(d). The mechanical quality factor of the fundamental mode, obtained from a Lorentzian fit to the data, is $Q_{01}=10$, whereas in the higher observed modes $Q_{11}=25$ and $Q_{21}=20$, respectively. On the one hand, higher resonant modes are favorable for mass sensing applications as higher $Q$ corresponds to a better resolution [16]. On the other hand, in higher modes the motion of the plate is not centrosymmetric, and the resonant measurement is thus far more sensitive to the laser spot positioning and drifts in the system, thus making correct analysis of the results more complex. Here we have chosen to work with the modes (11) and (21) for liquid mass sensing. 
2.3. Mass Sensing Model. Upon addition of mass to the bare microplate, in dynamic mode the resonance frequency of the device is altered. Equation (3) can be rewritten in terms of the total microplate mass $m$ :

$$
f_{m n}=\frac{\alpha_{m n}}{2} \sqrt{\frac{Y \varepsilon h}{\pi m}}
$$

where $h$ is the device thickness and $m=\pi a^{2} h \rho$ is the total mass. Upon addition of another layer (a bimorph) to the plate, the physical parameters that could vary are the effective Young modulus, strain, and mass. To the first order, the frequency can be expanded in all quantities:

$$
f=f_{0}+\frac{\partial f}{\partial m} \Delta m+\frac{\partial f}{\partial Y} \Delta Y+\frac{\partial f}{\partial \varepsilon} \Delta \varepsilon .
$$

Using (4) in this expression, the explicit frequency change becomes

$$
\begin{aligned}
\Delta f & =f_{0}-f \\
& =\frac{\alpha_{m, n}}{2 \sqrt{\pi}}\left\{\frac{1}{2} \sqrt{\frac{Y \varepsilon h}{m^{3}}} \Delta m-\frac{1}{2} \sqrt{\frac{\varepsilon h}{Y m}} \Delta Y-\frac{1}{2} \sqrt{\frac{Y h}{m \varepsilon}} \Delta \varepsilon\right\}
\end{aligned}
$$

and the relative frequency change reads:

$$
\frac{\Delta f}{f}=\frac{1}{2} \frac{\Delta m}{m}-\frac{1}{2} \frac{\Delta Y_{t}}{Y_{t}}-\frac{1}{2} \frac{\Delta \varepsilon}{\varepsilon} .
$$

In addition, the basic relationship between Young's modulus and the strain is given by the definition $Y=S / \varepsilon$, where $S$ is the built-in stress. Therefore, an increase in Young's modulus is accompanied with a decrease in the strain and vice versa. The total combined effect will almost completely balance out in the frequency shift, whereas the addition of mass always causes a negative shift in (7). Also, the expected change in Young's modulus and the strain are expected to be very small, and we take both to be up to $2 \%$, whereas the added mass is not constrained and is thus taken up to $10 \%$. In Figure 3 we have calculated, using (5)-(7) and Si parameters, the relative frequency shifts expected from individual changes in $Y$ (Figure 3(a)), in $\varepsilon$ (Figure 3(b)) and in $m$ (Figure 3(c)). It is thus expected that only the latter will strongly affect the resonant frequency.

Following this analysis, we henceforth assume that added mass is the significant effect that induces change in the plate resonant frequency. In this simple model, it is assumed that the added liquid is distributed homogenously on the plate surface. As the elastic properties of the plate and deposited liquid are taken to be constant, only the first term in (7) is considered to significantly contribute to frequency shifts observed experimentally.

\section{Results and Discussion}

In the experiment, we have monitored the resonant response spectrum of the microplate (Figure 1) as different liquids are added, in situ, on the top surface. Durable and repeatable operation has been observed using volatile liquids, namely, water and ethanol, which we present here. The plate was placed in a vertical alignment (see Figure 1(a)) and the chip containing a wide variety of different microresonators (including microplates, cantilevers, and bridges) has been attached to the piezoelectric crystal. A function generator is connected to the piezoelectric actuator to generate vibrations in the $10-30 \mathrm{KHz}$ ac frequency range. The whole chip therefore vibrated at the same frequency as the piezoelectric actuator, while the signal was swept over a span of interest. In the sensing experiment, we have focused the frequency sweep around modes of interest (namely, either around $\sim 20 \mathrm{KHz}$ for mode (11) or around $\sim 26 \mathrm{KHz}$ for mode (21)). Resonator motion was monitored by a HeNe laser beam reflected off the resonator and the Nickel film backplane, as the interference between these two reflections changes during the resonator motion, modulating the total reflected light intensity results. These changes are monitored by a fast photodetector signal through a spectrum analyzer, as detailed in Section 2. Starting from the baseline level of a dry device (corresponding to Figure 2(a)), we expose the plate to either droplet (Figure 2(b)) or spray (Figure 2(c)) liquid environment. In addition to the spectral response, we also monitor the maximum response resonant frequency in intervals of 10$60 \mathrm{~s}$.

3.1. Liquid Drip Resonant Sensing. In the first experiment, we have activated the piezoelectric actuator in the $18-24 \mathrm{kHz}$ frequency range. During vibration of the microplate, we have applied a single load of harsh water droplet. Figure 4(a) shows the response spectrum before and immediately after application of the droplet, whereas Figure 4(b) shows how the peak resonant frequency of the (21) mode changes with time. It is evident that the sudden decrease of the resonant frequency coincides with the water dripping event, followed by a slow recovery back to the original resonant value following full water evaporation.

3.2. Liquid Spray Resonant Sensing. Now instead of drops we apply a sprinkler to spray ethanol on the plate (see Figure 1(a)). This experiment lasted for considerably longer time and consisted of a series of 4 spray loads applied. Figure 5(a) shows the response spectrum before the experiment began and during application of five ethanol splashes, whereas Figure 5(b) shows how the peak resonant frequency of the (11) mode changes with time. Two splashes (at $t=$ $1000 \mathrm{~s}$ in Figure 5(b)) of ethanol spray follow three splashes (at $t=2000 \mathrm{~s}$ ) and four splashes (at $t=4000 \mathrm{~s}$ ). In this experiment the final quantity of liquid on the plate has been smaller than the experiment with water, as expected from spray rather than bulk droplet environment.

We note that after the experiment was done, the resonant frequency of the plate did not return to the original peak frequency observed before application of ethanol spray (see Figure 5(b)), even after long periods (days to several months). We estimate that the cause of this behavior, never observed with exposure to drip environments (such as in Figure 4), is the emergence of contaminating impurities dissolved within 


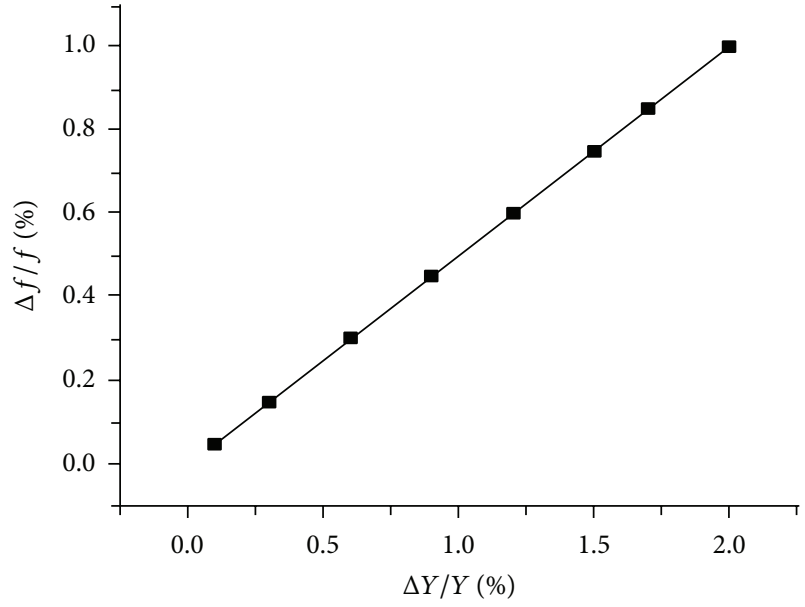

(a)

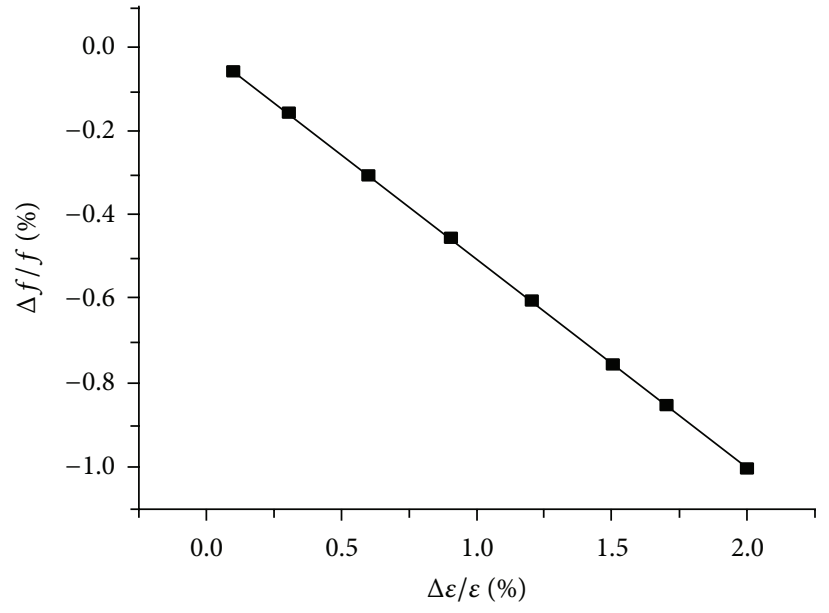

(b)

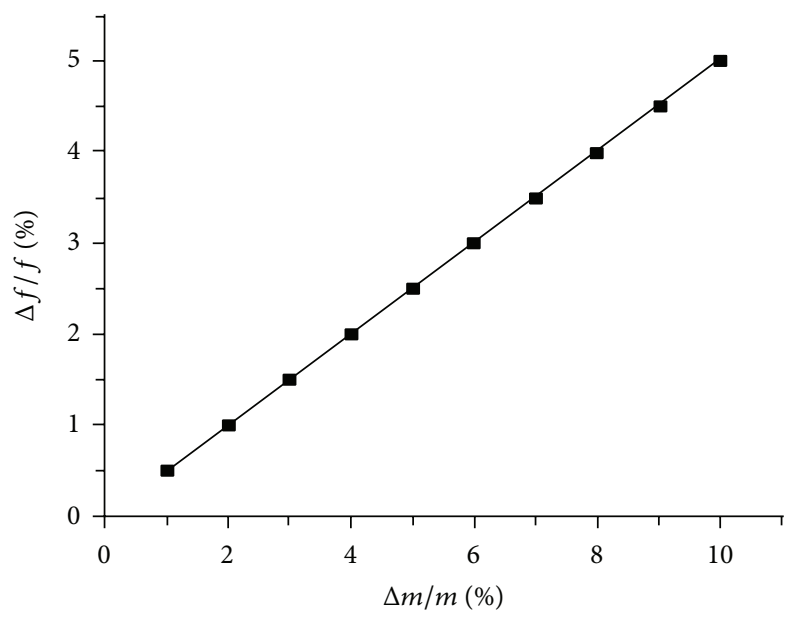

(c)

Figure 3: Relative change of resonant frequency, in percentage, as function of individual relative change in (a) Young's modulus, (b) strain, and (c) mass of the added liquid.

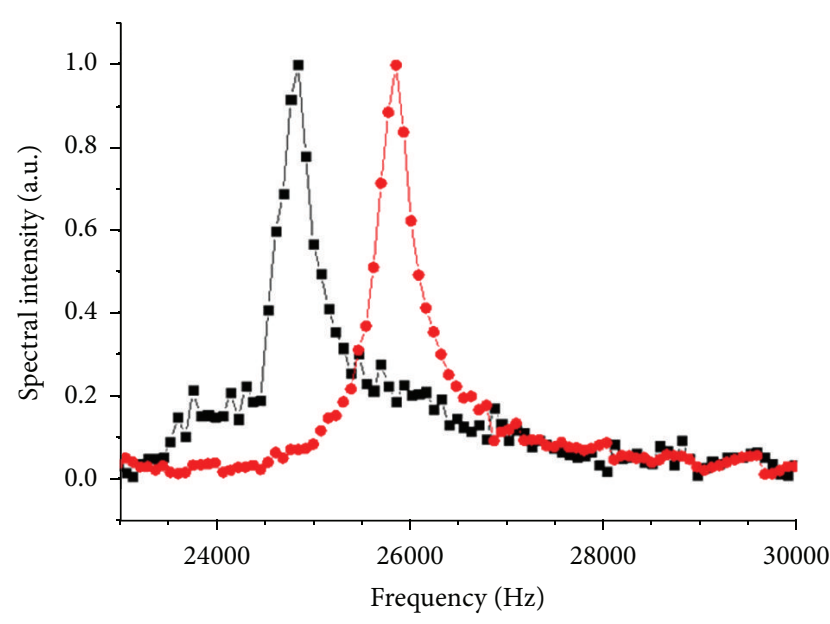

(a)

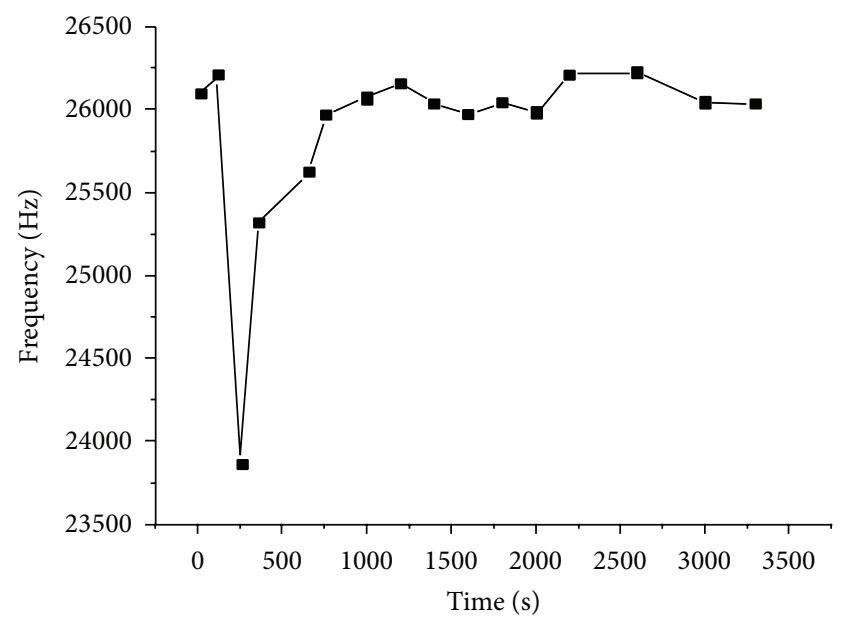

(b)

Figure 4: Measured data for liquid drop sensing: (a) frequency response around the $26 \mathrm{kHz}$ (21) resonance before (red circles) and after (black squares) water drip. (b) Temporal monitoring of the corresponding central peak frequency as a function of time during and after the water dripping on the microplate. 


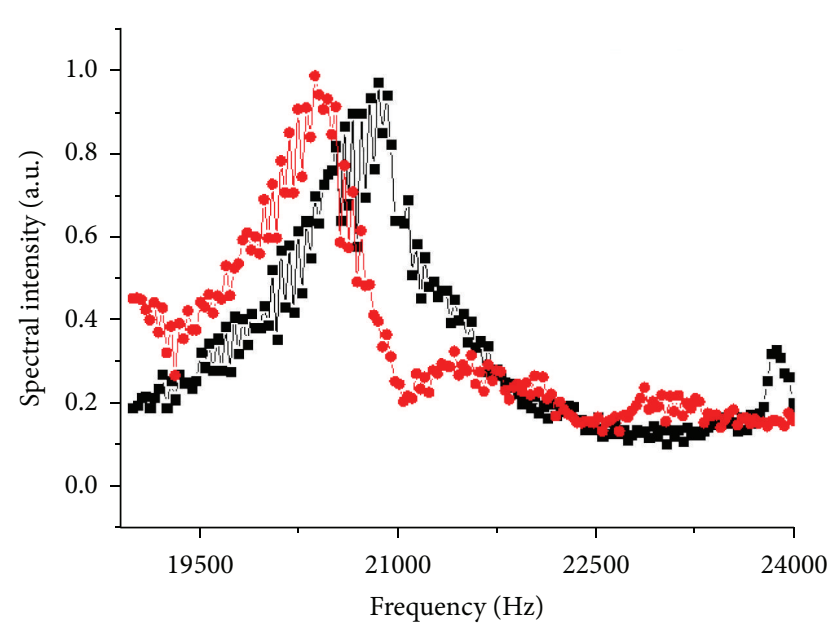

(a)

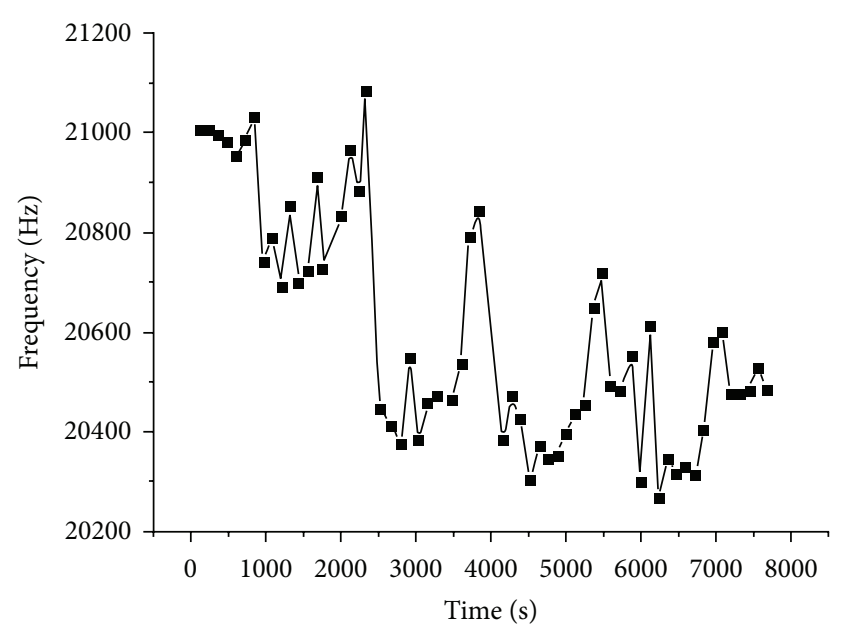

(b)

FIGURE 5: Measured data for liquid spray sensing: (a) shift in mode (11) spectrum due to ethanol spray. (b) Change in the peak resonant frequency monitored as a function of time during ethanol spraying session.

the applied spray, as these do not evaporate and remain on the plate surface as residual sediments (see Figure 2(c)).

\subsection{Added Mass Estimation and Ultimate Device Resolution.} In Figure 6, we estimate the amount of added mass from the measured frequency shifts, using the model in Section 2.3. Figure 6(a) shows the amount of liquid on the plate as a function of the frequency for both water drip (peak $26 \mathrm{kHz}$ ) and ethanol spray (peak $21 \mathrm{kHz}$ ). The linear relationship between the mass of liquid and the frequency change is consistent with the theoretical formula (7). As expected, the slope of the curve describing the shift in peak $26 \mathrm{kHz}$ is greater than the corresponding slope for peak $21 \mathrm{kHz}$, consistent with the theoretical estimation. The coefficients $\alpha_{m n}$ in (3) used in the estimation are $\alpha_{21}=5.135$ and $\alpha_{11}=$ 3.832 for the modes (21) and (11), respectively.

Figures 6(b) and 6(c) show the water and ethanol amount, respectively, on the plate as a function of time. The amount of water and ethanol on the plate was calculated using the theoretical formula (11).

We emphasize again that the elastic properties of the plate are assumed to be unaffected by the deposition of liquid. The change in frequency given by the equation above will therefore depend mostly on the change in the plate mass, which is the only free parameter. From (7) the frequency is inversely proportional to the mass square root $f \propto 1 / \sqrt{m}$. Therefore, the initial frequency with no added mass is $f_{0} \propto$ $1 / \sqrt{m_{0}}$, where $m_{0}$ is the free mass of the unloaded plate. If mass $\Delta M$ of liquid is added to the plate the resonant frequency will change according to $f \propto 1 / \sqrt{m_{0}+\Delta M}$.

The liquid mass $\Delta M$ can be written as a function of the frequency ratio

$$
\frac{f_{0}}{f}=\sqrt{1+\frac{\Delta M}{m_{0}}} \Longrightarrow \Delta M=m_{0}\left(\frac{f_{0}}{f}\right)^{2}-m_{0}
$$

If in addition the added mass is assumed to be small in comparison to the original plate mass, that is, $\Delta M<<m_{0}$, we get a ratio $f_{0} / f=1+(1 / 2)\left(\Delta M / m_{0}\right)$ and thus

$$
\Delta M=2 m_{0} \frac{f_{0}}{f}-2 m_{0} .
$$

The relation between the change in frequency and the change in mass can be given, under this simplest model, as

$$
\begin{aligned}
f_{0}-f & =\frac{\beta}{\sqrt{m_{0}}}-\frac{\beta}{\sqrt{m_{0}+\Delta M}} \\
& =\frac{\beta}{\sqrt{m_{0}}}\left\{1-\frac{1}{\sqrt{1+\Delta M / m_{0}}}\right\},
\end{aligned}
$$

where $\beta=\left(\alpha_{m n} / 2 \pi a\right) \sqrt{Y V \varepsilon}$ is a constant which depends only on the plate characteristics (see Section 2.2).

If in addition $\Delta M<<m_{0}$, one gets

$$
\Delta f \sim \frac{\beta}{\sqrt{m_{0}}}\left\{1-\left(1-\frac{1}{2} \frac{\Delta M}{m_{0}}\right)\right\}=\frac{\beta}{2 m_{0}^{3 / 2}} \Delta M
$$

and a linear dependence between the change in mass and the change in the frequency exists (Figure 6). In comparison with the limit of mass sensing using gravimetric mechanical resonators [14], (11) coincides with the analysis, whereas (8) is generally valid, also under harsh environmental conditions. In the former limit, the minimum detectable mass in our plate is estimated as $\Delta M \sim 20 \mathrm{ng}$, but here other physical effects might become important, such as thermomechanical coupling [14].

\section{Conclusions and Outlook}

In this work we have used a single-layer free-standing polycrystalline silicon microplate resonator for the detection 


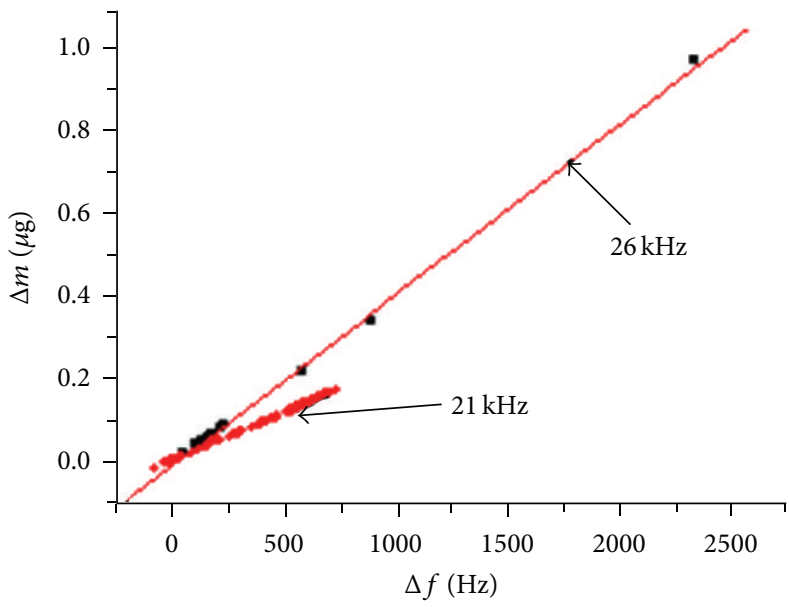

(a)

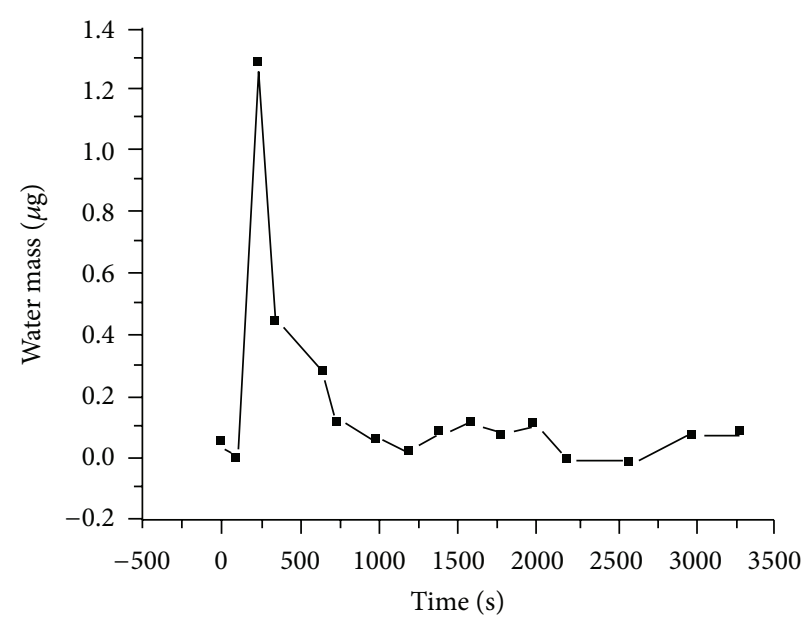

(b)

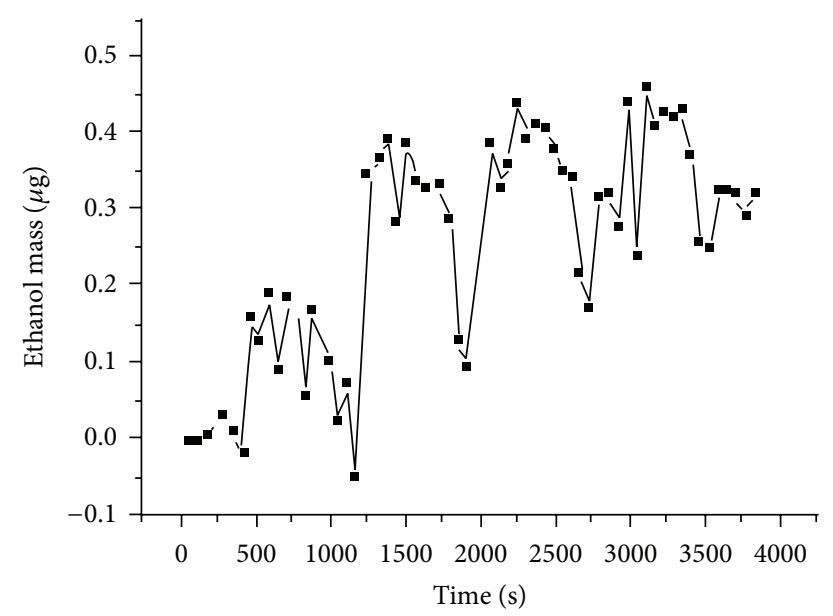

(c)

Figure 6: (a) Mass of the liquid (water/ethanol) deposited on the plate as a function of the shift in resonant frequency, for both (11) and (21) modes. (b) Estimated mass of water on the plate as a function of time, for water drip (Figure 4). (c) Mass of ethanol on the plate as a function of time, for ethanol spray (Figure 5).

of added liquid masses and attained sustainable operation of the plate under harsh wet droplet and spray conditions. Mass spectroscopy using the microplate with an electrooptical detection setup that is synchronized in the frequency domain with mechanical vibration modes was obtained over long stability periods. With the available instrumentation, the resonant response spectrum of the plate has been monitored as different liquids were added, in situ, on the device surface, and sustainable mass spectroscopy was achieved under harsh loading conditions. A simplified analysis indicated large ( 0.1-1 micrograms) mass loading attained over a $\sim 200$ nanogram plate.

We plan to extend the experiment to multiplexed mass spectroscopies of different analyte mixtures in liquid, as well as gaseous, environments. Despite the robustness of the device under harsh liquid environment, in the lower limit of mass spectroscopy the current experiment has shown a minimum mass detection resolution of only $\sim 20$ nanograms, which is not sufficiently sensitive for selective vapor mass spectroscopy. The current sensor capabilities are not limited by the electrooptical setup but rather result from relatively low quality factors exhibited by the microplate (10-25), which result from ambient air friction combined with the low operation frequencies. The latter restrictions can be improved with optimized device geometries and new driving mechanisms.

\section{Conflict of Interests}

The authors declare that there is no conflict of interests regarding the publication of this paper.

\section{Acknowledgments}

This research was partially supported by the Ort Braude College under Grants 5000.838.3-64 and 5000.838.3-65. Yoav Linzon and Dafne Guetta thank the College active researchers 
scholarship. The authors thank Assaf Yaakobovitz for fabrication of the devices and Dr. Eli Raz, Head of the Optical Engineering Department, for his dedicated support of the project.

\section{References}

[1] W. E. Newell, "Miniaturization of tuning forks," Science, vol. 161, no. 3848 , pp. 1320-1326, 1968.

[2] B. Ilic, H. G. Craighead, S. Krylov, W. Senaratne, C. Ober, and P. Neuzil, "Attogram detection using nanoelectromechanical oscillators," Journal of Applied Physics, vol. 95, no. 7, pp. 36943703, 2004.

[3] M. S. Hanay, S. Kelber, A. K. Naik et al., "Single-protein nanomechanical mass spectrometry in real time," Nature Nanotechnology, vol. 7, no. 9, pp. 602-608, 2012.

[4] K. Jensen, K. Kim, and A. Zettl, "An atomic-resolution nanomechanical mass sensor," Nature Nanotechnology, vol. 3, no. 9, pp. 533-537, 2008.

[5] R. K. Burra, J. Vankara, and D. V. Reddy Rama Kota, "Effect of added mass using resonant peak shifting technique," Journal of Micro/Nanolithography, MEMS, and MOEMS, vol. 11, no. 2, Article ID 021203, 2012.

[6] M. Li, E. B. Myers, H. X. Tang et al., "Nanoelectromechanical resonator arrays for ultrafast, gas-phase chromatographic chemical analysis," Nano Letters, vol. 10, no. 10, pp. 3899-3903, 2010.

[7] D. R. Southworth, L. M. Bellan, Y. Linzon, H. G. Craighead, and J. M. Parpia, "Stress-based vapor sensing using resonant microbridges," Applied Physics Letters, vol. 96, no. 16, Article ID 163503, 2010.

[8] H. J. Lee, K. K. Park, M. Kupnik, Ö. Oralkan, and B. T. KhuriYakub, "Chemical vapor detection using a capacitive micromachined ultrasonic transducer," Analytical Chemistry, vol. 83, no. 24, pp. 9314-9320, 2011.

[9] H. J. Lee, K. K. Park, M. Kupnik, N. A. Melosh, and B. T. KhuriYakub, "Mesoporous thin-film on highly-sensitive resonant chemical sensor for relative humidity and $\mathrm{CO}_{2}$ detection," Analytical Chemistry, vol. 84, no. 7, pp. 3063-3066, 2012.

[10] D. J. Joe, Y. Linzon, V. P. Adiga et al., "Stress-based resonant volatile gas microsensor operated near the critically buckled state," Journal of Applied Physics, vol. 111, no. 10, Article ID 104517, 2012.

[11] J. L. Arlett, E. B. Myers, and M. L. Roukes, "Comparative advantages of mechanical biosensors," Nature Nanotechnology, vol. 6, no. 4, pp. 203-215, 2011.

[12] R. A. Barton, B. Ilic, S. S. Verbridge, B. R. Cipriany, J. M. Parpia, and H. G. Craighead, "Fabrication of a nanomechanical mass sensor containing a nanofluidic channel," Nano Letters, vol. 10, no. 6, pp. 2058-2063, 2010.

[13] I. Bargatin, E. B. Myers, J. S. Aldridge et al., "Large-scale integration of nanoelectromechanical systems for gas sensing applications," Nano Letters, vol. 12, no. 3, pp. 1269-1274, 2012.

[14] K. L. Ekinci, Y. T. Yang, and M. L. Roukes, "Ultimate limits to inertial mass sensing based upon nanoelectromechanical systems," Journal of Applied Physics, vol. 95, no. 5, pp. 26822689, 2004.

[15] J. Chaste, A. Eichler, J. Moser, G. Ceballos, R. Rurali, and A. Bachtold, "A nanomechanical mass sensor with yoctogram resolution," Nature Nanotechnology, vol. 7, no. 5, pp. 301-304, 2012.
[16] M. Calleja, P. M. Kosaka, Á. San Paulo, and J. Tamayo, "Challenges for nanomechanical sensors in biological detection," Nanoscale, vol. 4, no. 16, pp. 4925-4938, 2012.

[17] Y. Linzon, S. Krylov, B. Ilic et al., "Real-time synchronous imaging of electromechanical resonator mode and equilibrium profiles," Optics Letters, vol. 35, no. 15, pp. 2654-2656, 2010.

[18] A. Rabinovich, A. Ya'akobovitz, and S. Krylov, "Fringing electrostatic field actuation of microplates for open air environment sensing," Journal of Vibration and Acoustics, vol. 136, no. 4, Article ID 041013, 2014.

[19] S. Timoshenko and D. H. Young, Vibration Problems in Engineering, D. Van Nostrand, Princeton, NJ, USA, 3rd edition, 1961.

[20] S. M. Hu, "Stress-related problems in silicon technology," Journal of Applied Physics, vol. 70, no. 6, pp. R53-R80, 1991. 

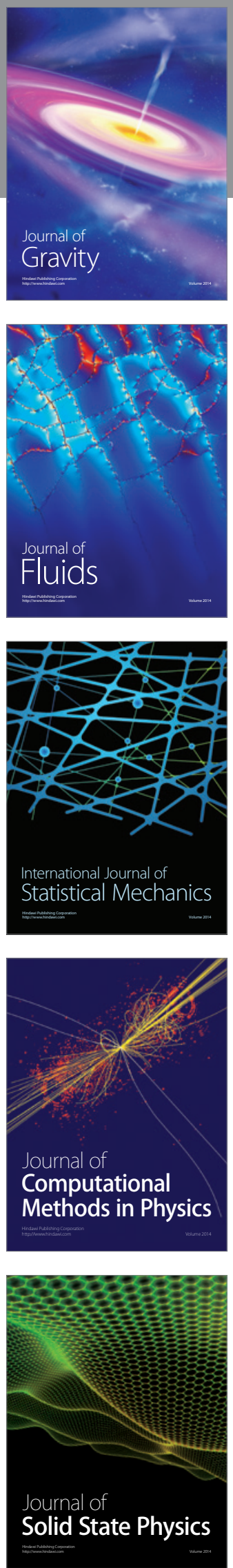

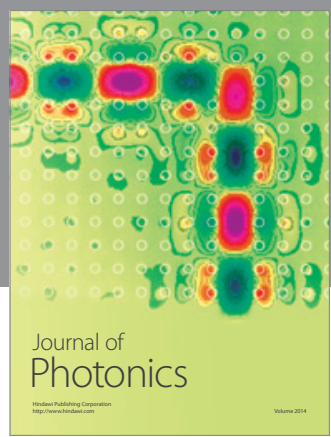

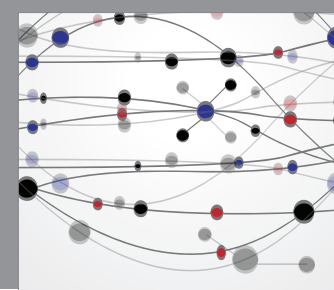

The Scientific World Journal

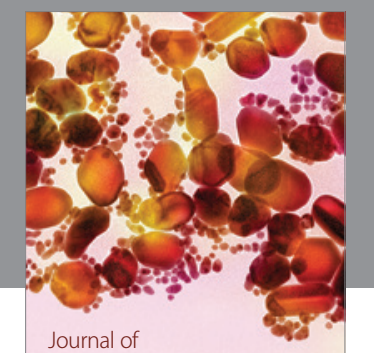

Soft Matter
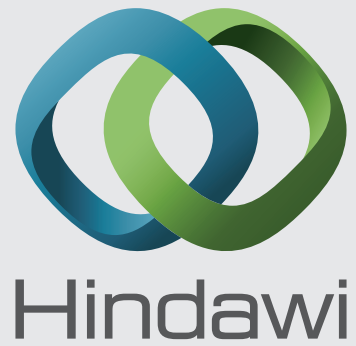

Submit your manuscripts at

http://www.hindawi.com
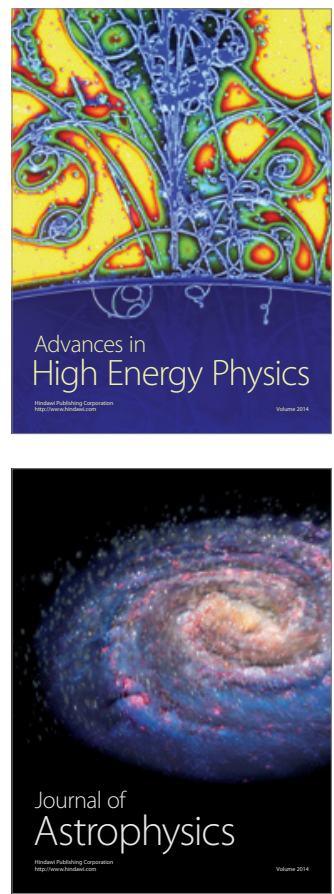
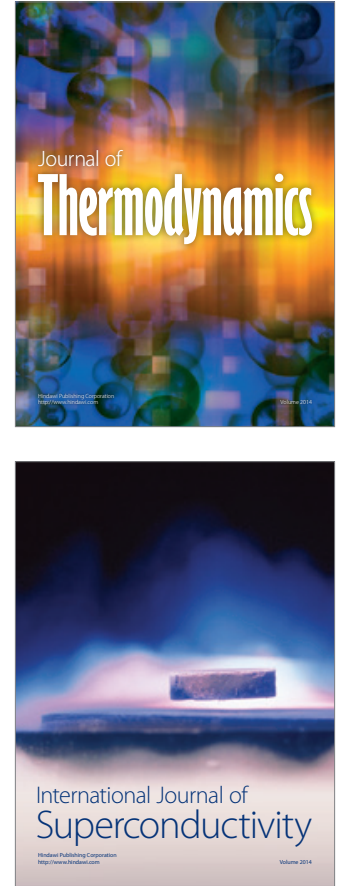
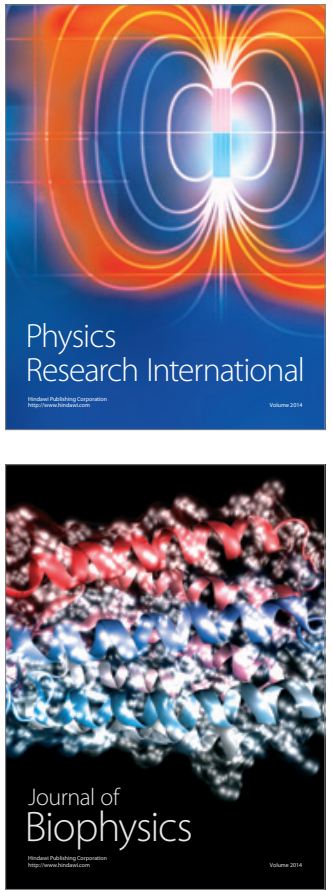
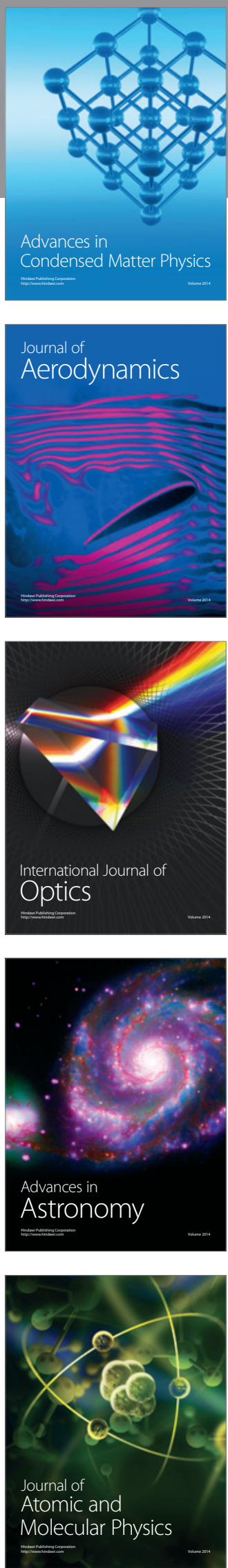